\title{
A retroperitoneal cyst with unusual urinary histogenesis: clinical and immunomorphological characteristics
}

This article was published in the following Dove Press journal:

International Medical Case Reports Journal

17 November 2010

Number of times this article has been viewed

\author{
Giovanni Branca' \\ Antonio leni' \\ Valeria Barresi' \\ Antonino Versaci ${ }^{2}$ \\ 'Section of Anatomic Pathology, \\ ${ }^{2}$ Section of General Surgery, \\ Department of Human Pathology, \\ Azienda Ospedaliera Universitaria \\ "Policlinico G. Martino", University \\ of Messina, Messina, Italy
}

Correspondence:Antonio leni Dipartimento di Patologia Umana, Policlinico Universitario G. Martino, Pad D, Via Consolare Valeria,

98। 25 Messina, Italy

Tel +39 902212543

Fax +39 902938324

Email calaienco@hotmail.com

\begin{abstract}
Primary retroperitoneal cysts are rare benign lesions which often present as an incidental radiological finding and also cause abdominal symptoms. A 47-year-old woman was admitted to the surgery unit because of right-sided abdominal pain. Computed tomographic scan investigation confirmed a circumscribed, oval, dishomogeneous mass in the right anterior pararenal space. The final diagnosis was obtained after laparotomy excision and pathological evaluation. Immunohistochemical profile was characterized by positive expression of epithelial cystic cells for CKAE1/AE3, CK7, CK8, and CK18, with EMA sustaining the diagnosis of a benign retroperitoneal cyst of urinary system origin.
\end{abstract}

Keywords: retroperitoneal cyst, cytokeratins, urinary tract

\section{Introduction}

Primary retroperitoneal cysts are rare with an estimated incidence of $1 / 5750$ to $1 / 250,000 .{ }^{1}$ They can appear at any age but have a peak of incidence in the fourth decade of life with female preponderance. ${ }^{2}$ Clinically, there are no pathognomonic signs or symptoms for retroperitoneal cysts, and in one-third of patients, the cyst is found incidentally. ${ }^{1}$ About two-thirds of patients present with an abdominal mass or chronic abdominal symptoms. ${ }^{1}$ Other symptoms include back pain, pain and/or edema of the lower limbs, dyspnea, and anorexia accompanied by weight loss or fever. Most of these cysts originate from vestiges of embryonic blastemas, and their inner lining is usually compatible with either a mesothelial or mesonephric origin, although in some instances the lining is of Müllerian type with either a serous or mucinous appearance. ${ }^{3}$

\section{Case report}

A 47-year-old woman was admitted to the surgery unit of Polyclinic Hospital "G Martino" (Messina, Italy) in October 2009 because of right-sided abdominal pain which had been present for $5 \mathrm{~h}$. Abdominal examination revealed vague pain localized in the right upper quadrant and in epigastric and periumbilical regions with moderate resistance. Laboratory examinations were all within normal limits.

Abdominal ultrasonography revealed a fluid collection mass (measuring $12 \mathrm{~cm}$ of maximum diameter) localized in the upper right abdomen and in contact with right renal hilus.

Computerized tomography imaging of the abdomen showed a circumscribed, oval, dishomogeneous mass $(8.5 \times 5 \times 5.5 \mathrm{~cm})$ in the right anterior pararenal space, anterior to the right kidney, lateral to the second portion of the duodenum, inferior to the right 
lobe of the liver, displacing and compressing the hepatic portal vein and the inferior vena cava (Figure 1).

At laparatomy, the cyst was found to be retroperitoneal, situated behind the mesentery of the hepatic flexure of the colon. The cyst compressed the liver, the gallbladder, and hepatic flexure of the colon anteriorly. Macroscopically, the mass was a pouch-like structure with a hard-elastic consistency containing hematic fluid. Careful and complete total surgical removal of the cyst was performed.

Gross examination of the specimen showed a collapsed, previously opened, dark gray to brown colored, unilocular, thin-walled cyst measuring $40 \times 60 \mathrm{~mm}$ (Figure 2). The inner lining was mostly smooth. The entire cyst was sectioned and submitted for microscopic examination.

The surgical specimen was fixed in $4 \%$ formaldehyde, completely sampled and routinely processed. Paraffin sections were stained with hematoxylin and eosin.

Immunohistochemical staining was performed using antibodies against BCL2 (1:100 DAKO), CD10 (1:80 DAKO), CK AE1/AE3 (1:50 DAKO), CK7 (1:100 DAKO), CK8 (1:50 DAKO), CK18 (1:50 DAKO), CK20 (1:50 DAKO), EMA (1:1000 DAKO), calretinin (1:100 DAKO), podoplanin (D2-40) (1:200 DAKO), estrogen (1:35 DAKO) and progesterone (1:50 DAKO) receptors, CD34 (1:50 DAKO), CD31 (1:40 DAKO), and CA125 (1:20 DAKO).

Histologically, the cyst was found to be lined with cuboidal epithelium. There was no cytological atypia or malignancy in the lining epithelium or stromal tissue components. The cyst wall consisted of a thin layer of fibrous tissue which showed areas of chronic inflammation and subepithelial vascular proliferation.

The results of immunohistochemical analysis of the epithelium lining of the cyst are summarized in Table 1. In particular, immunohistochemistry showed diffused strong cytoplasmic staining for CKAE1/AE3 antibodies. The epithelial cystic cells were immunoreactive to CK8 and CK18, while the CK7 antibody did not show diffused cytoplasmic staining. EMA staining was diffusely and strongly localized at the cell membrane. The markers for the remaining antigens tested (BCL2, CK20, calretinin, podoplanin (D2-40), CD10, CD31, CD34, CA125, ER, PGR) were negative.

\section{Discussion}

Retroperitoneal cysts have been defined by Handfield Jones as those cysts lying in the retroperitoneal fatty tissues which have no connection with any adult anatomic structure except the areolar tissue. ${ }^{4}$ Epithelium-lined retroperitoneal cysts can be categorized on an embryological and histogenetic basis as bronchogenic cysts, lymphatic cysts, mesothelial cysts, enteric cysts, and urogenital cysts. ${ }^{2,5,6}$

Bronchogenic cysts result from abnormal budding of the developing tracheobronchial tree, with separation of the buds from the normal airways during the third to seventh weeks of development. ${ }^{3-6}$ They are usually found around the
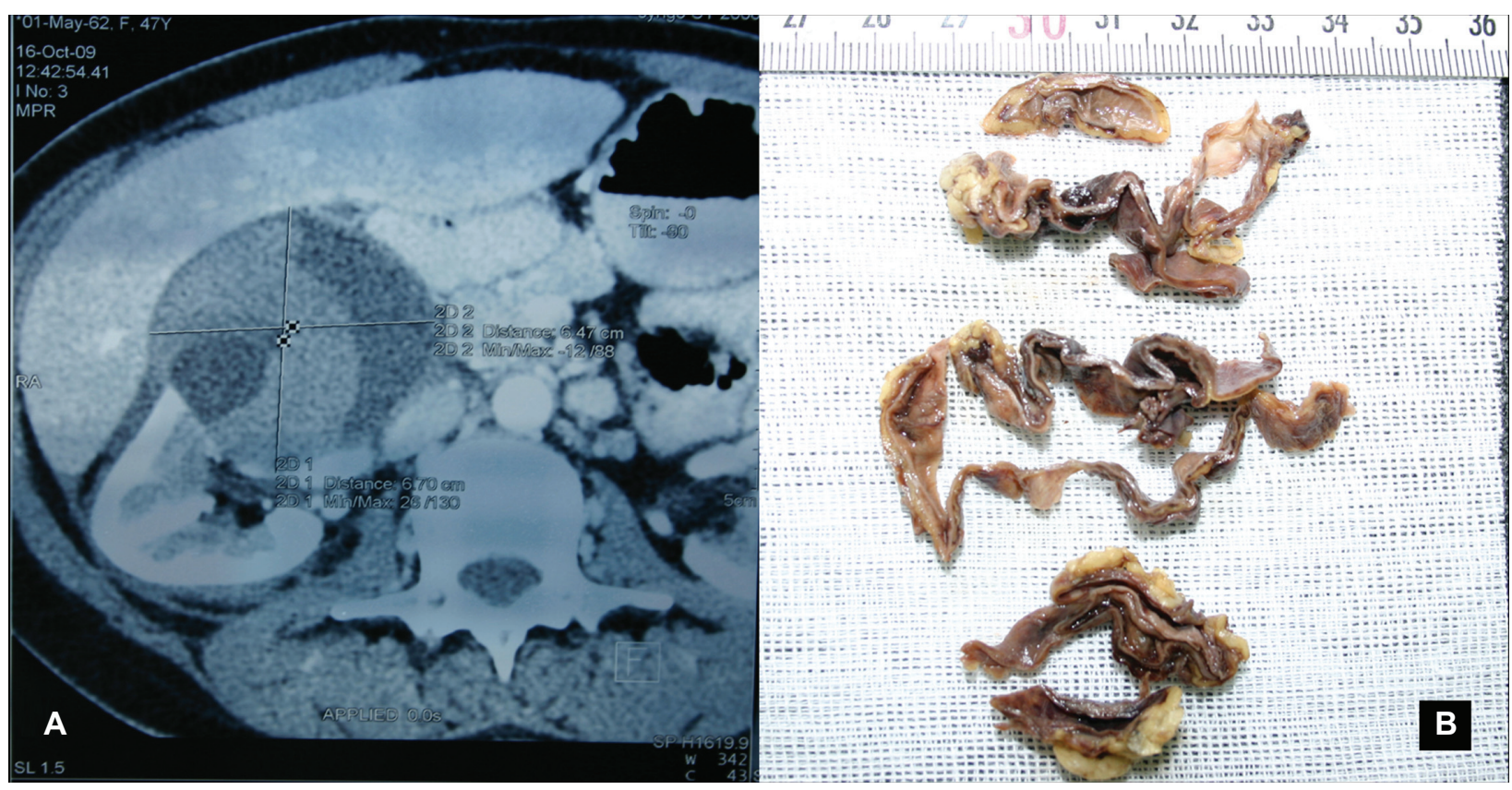

Figure I A) Computed tomography of the abdomen shows a retroperitoneal mass in the right anterior pararenal space (transverse cross section). B) Photograph of the gross specimen shows the cyst wall. 

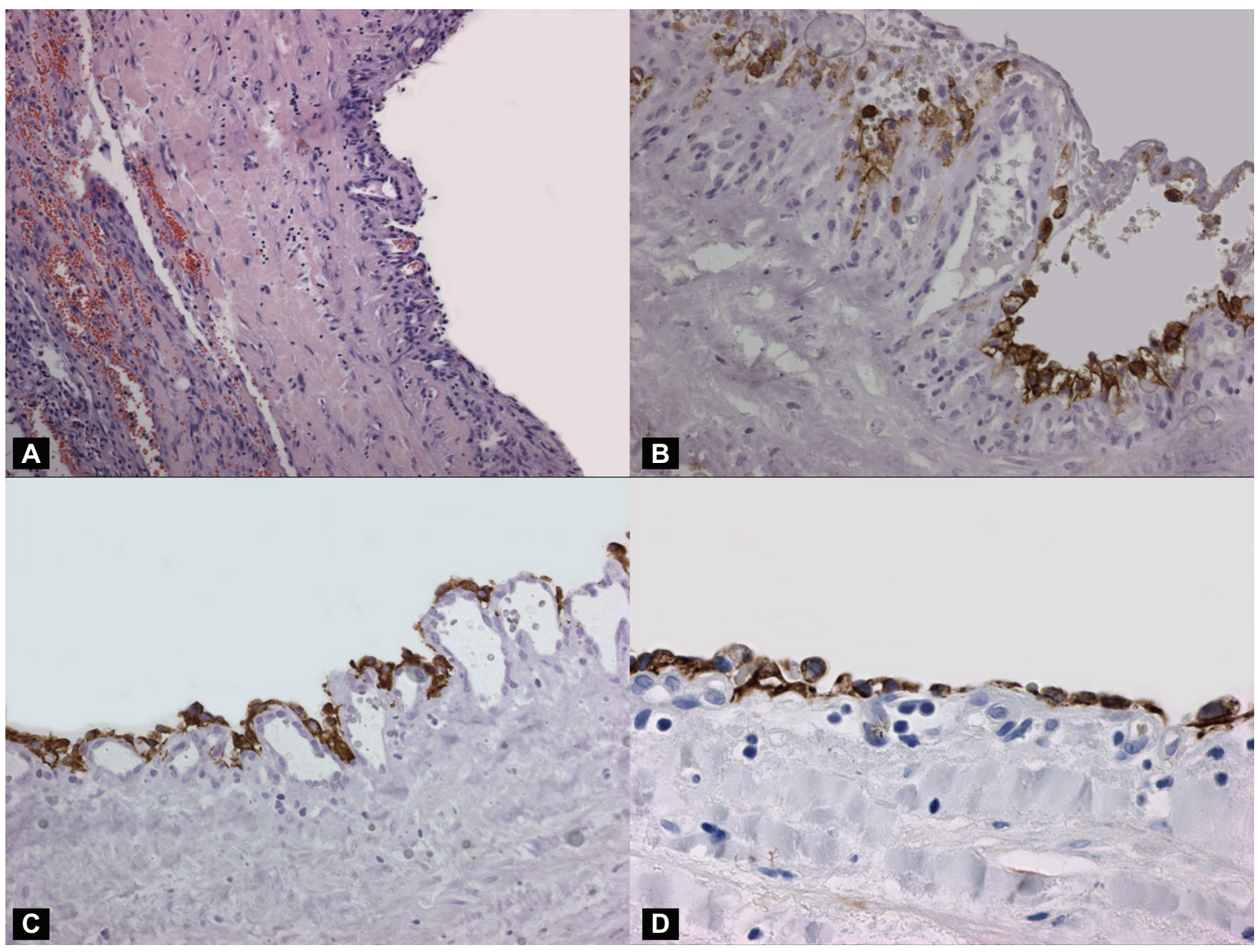

Figure 2 A) The cystic wall was lined by cuboidal epithelial cells (H\&E stain, 80×). B) The epithelial cells were immunoreactive with EMA (EMA stain; original magnification, I60X). C) A strong immunopositivity was found also with CK AEI/AE3 (CK AEI/AE3 stain; original magnification, 80x). D) CKI8 revealed an evident immunostaining in cuboidal epithelial cells (D) (CK AEI/AE3 stain; original magnification, I60×).

Abbreviation: H\&E, hematoxylin and eosin.

adrenal gland and may simulate a primary adrenal neoplasm. ${ }^{5}$ Bronchogenic cysts are lined by respiratory epithelium with bronchial seromucous glands, smooth muscle, and nodules of hyaline cartilage. ${ }^{3-6}$ Lymphatic cysts can be unilocular or multilocular containing clear or milky fluid and lined with a single layer of flattened endothelium. ${ }^{6}$ They occur due to failure of the developing lymphatic tissue to establish normal communication with the remainder of the lymphatic system. ${ }^{6}$ Lymphatic cysts are more common in men and can occur at any age. ${ }^{6}$ They are subdivided into those formed in the lymphatic cysts returning from the intestine, known as chylous cysts, and those arising in the lymphatic field behind the peritoneum, not connected with the intestine, and analogous in their origin to the single cystic lymphangioma seen in the head and neck. ${ }^{1}$

Solitary cysts have a translucent wall, watery fluid in the lumen, and a lining composed of one or more layers of mesothelial cells. ${ }^{5}$ They probably represent acquired inclusion cysts related to chronic inflammation. ${ }^{5}$ A probably related condition has been designated cystic or multicystic benign mesothelioma. Grossly multiple thin-walled cysts containing watery secretion are present and measure up to $15 \mathrm{~cm}$ or more in diameter. ${ }^{6}$ Microscopically, the cysts are lined by flattened or cuboidal mesothelial cells. ${ }^{5}$ When flat, the cells closely simulate the appearance of endothelial cells. The mesothelial cells react immunohistochemically for keratin and calretinin, ${ }^{5}$ are negative for FVIII-related antigen and other endothelial markers, ${ }^{5}$ and sometimes show focal reactivity for hormone receptors. ${ }^{5}$ The wall, which is devoid of smooth muscle, usually shows foci of chronic inflammation, hemorrhage, and fibrin deposition. ${ }^{5}$ Cystic mesothelioma is not related to prior asbestos exposure. It does not metastasize but may recur locally and occurs more frequently in women. ${ }^{6}$

Enteric cysts are rare congenital multicystic lesions that arise from vestiges of the embryonic hindgut and occur between the rectum and sacrum, commonly in middle-aged 
Table I Immunophenotype of the retroperitoneal cyst in the present study

\begin{tabular}{ll}
\hline Antibody & Staining \\
\hline EMA & + \\
CK AEI/AE3 & + \\
CK7 & + \\
CK8 & + \\
CK18 & + \\
CK20 & - \\
CD3I & - \\
CD34 & - \\
CAI25 & - \\
Calretinin & - \\
Podoplanin (D2-40) & - \\
ER & - \\
PGR & - \\
CDI0 & - \\
BCL2 & - \\
\hline
\end{tabular}

Notes: +, positive staining; -, negative staining.

women. ${ }^{6}$ Lordon et al described the pathological criteria for diagnosis of these cysts. These criteria include the presence of alimentary mucous membrane lining a smooth muscle coat and intimate attachment to some part of the gastrointestinal tract. ${ }^{7}$ Malignant degeneration of enteric cyst has also been reported and results in adenocarcinoma. ${ }^{6,7}$ Urogenital cysts can be subclassified into pronephric, mesonephric, metanephric, and Müllerian types. ${ }^{1,5,6}$ The majority of urogenital cysts occur near the kidney. They arise from the vestiges of the embryonic urogenital apparatus. When opened, they have a smooth, glistening lining membrane, are unilocular, and contain a clear serous fluid. Histologically, the cyst wall is thin, consisting of a cellular fibrous tissue lined by a low columnar epithelium, or cubical and rarely flattened. ${ }^{1}$ Occasionally, they display histologic features indicative of a mesonephric or metanephric origin. In particular, among the urogenital cysts, those with positive immunohistochemical expression for CD10 and BCL2 antibodies in epithelial cells are classified as mesonephric, evidencing an origin from mesonephric remmants ${ }^{8-10}$ and those containing nephric duct and metanephrogenic mesenchyme are classified as metanephric. Cysts of Müllerian origin are extremely rare in the retroperitoneum and occur in women aged from 19 to 47 years. ${ }^{6}$ They result from persistence of Müllerian ducts derivatives, ${ }^{5}$ which might have the capacity to grow in later life under the influence of abnormal hormonal stimuli. ${ }^{2-6}$ Histologically, those cysts are lined by fallopian tube-type epithelium (cuboidal to columnar with cilia), and the cyst wall consists of thicker smooth muscle. ${ }^{5,6}$ Clinical history may be useful for differentiating Müllerian cyst from other retroperitoneal cysts because the former usually occurs in obese patients with menstrual irregularities. ${ }^{5}$

In our case, a mesothelial nature of the cyst was excluded by negative immunohistochemical expression of calretinin and podoplanin (D2-40), while the hypothesis of lymphatic origin was excluded by the absence of endothelium antigens CD31 and CD34 as well as by the negative staining for podoplanin (D2-40). Moreover, the immunohistochemical absence of CA125, estrogen, and progesterone receptors ruled out the possibility of Müllerian origin of our observation.

However, the histological features and immunohistochemical profile characterized by positive expression of CKAE1/AE3, CK7, CK8, CK18, EMA, and negative staining for CK20 strongly supported the epithelial nature of cuboidal cystic cells and also the diagnosis of a benign retroperitoneal cyst of urinary system origin. ${ }^{11}$

Specifically, the retroperitoneal cyst observed in the present case may be classified as pronephric, since one diagnosis should be confirmed by: negative immunohistochemical staining for CD10 and BCL2 in epithelial cystic cells, ${ }^{8-10}$ positive immunoreactivity for CK $18,{ }^{12}$ and the histological absence of renal tissue within cystic wall. In particular, either immunohistochemistry or histomorphological aspects could exclude both the mesonephric and metanephric nature of the present case.

\section{Disclosure}

The authors report no conflicts of interest in this work.

\section{References}

1. Alzaraa A, Mousa H, Dickens P, Allen J, Benhamida A. Idiopathic benign retroperitoneal cyst: a case report. J Med Case Reports. 2008;2: 43.

2. Yohendran J, Dias MM, Eckstein R, Wilson T. Benign retroperitoneal cyst of Mullerian type. Asian J Surg. 2004;27(4):333-335.

3. Haddadin WJ, Reid R, Jindal RM. A retroperitoneal bronchogenic cyst: a rare cause of a mass in the adrenal region. J Clin Pathol. 2001; 54(10):801-802.

4. Handfield-Jones RM. Retroperitoneal cyst, their pathology, diagnosis and treatment. Brit J Surg. 1924;12:119-134.

5. Rosai J. Peritoneum, retroperitoneum and related subjects. Ackerman's Surgical Pathology. St Louis, MO: Mosby; 2004:2375-2393.

6. Yang DM, Jung DH, Kim H, et al. Retroperitoneal cystic masses: $\mathrm{CT}$, clinical, and pathologic findings and literature review. Radiographics. 2004;24(5):1353-1365.

7. Lordan JT, Jones RL, Karanjia ND, et al. A rare case of a retroperitoneal enterogenous cyst with in-situ adenocarcinoma. World J Surg Oncol. 2007;5:113.

8. Oliva E. CD10 expression in the female genital tract: does it have useful diagnostic applications? Adv Anat Pathol. 2004;11(6):310-315.

9. Pavlakis K, Messini I, Yiannou P, et al. A pre-tailored panel of antibodies in the study of cervical mesonephric remnants. Gynecol Oncol. 2010;116(3):468-472. 
10. Ordi J, Romagosa C, Tavassoli FA, et al. CD10 expression in epithelial tissues and tumors of the gynecologic tract: a useful marker in the diagnosis of mesonephric, trophoblastic, and clear cell tumors. Am J Surg Pathol. 2003;27(2):178-186.

11. Alonso A, Ikinger U, Kartenbeck J. Staining patterns of keratins in the human urinary tract. Histol Histopathol. 2009;24(11):1425-1437.
12. Wang YH, Chen YH, Lin YJ, Tsai HJ. Spatiotemporal expression of zebrafish keratin 18 during early embryogenesis and the establishment of a keratin 18:RFP transgenic line. Gene Expr Patterns. 2006;6(4): $335-339$.

\section{Publish your work in this journal}

The International Medical Case Reports Journal is an international, peer-reviewed open-access journal publishing original case reports from all medical specialties. Previously unpublished medical posters are also accepted relating to any area of clinical or preclinical science. Submissions should not normally exceed 2,000 words or
4 published pages including figures, diagrams and references. The manuscript management system is completely online and includes a very quick and fair peer-review system, which is all easy to use. Visit http://www.dovepress.com/testimonials.php to read real quotes from published authors.

Submit your manuscript here: http://www.dovepress.com/international-medical-case-reports-journal-journal 